\title{
Consumer informatics and health information on obesity
}

\begin{abstract}
The use of internet by the consumer for health information on obesity, weight management and other obesity related diseases continues to soar. The need for information has formed a health informatics sub-specialty referred to as Consumer Health Informatics. Obesity is just one of many health conditions where consumer education for empowerment is important to improve their own health. In 2012, the percentage of obese adults 18 and older was $27.7 \%$ in US nationally. Unfortunately, by 2014 the US percentage of obese adults increased to $28.9 \%$. The obesity trends in Canada are similar to those of the United States. In 2012, Statistics Canada has reported that $18.4 \%$ of Canadians aged 18 and older have been reported as being obese. The most recent Canadian statistics from 2014 has reported 20.2\% of Canadians aged 18 and older as obese.

In today's technological society there are additional new abilities and skills required for health literacy. Computer literacy skill is required to assess, understand, and apply health information obtained through the internet [5]. Being able to assess the information as to its reliability and accuracy is critical vs. assessing the sophisticated design of any given webpage. In my opinion, in effort to assist consumers searching for health in regards to health literacy and computer literacy skills the US government should promote and place more awareness on health information websites. Therefore, my recommendation for consumers is to follow the following steps by visiting ".gov", ".edu". and ".org" sites first prior to searching the less reliable and accurate ".com" websites for health information online.
\end{abstract}

Keywords: consumer informatics, health information online, health literacy, computer literacy, obesity
Volume 4 Issue 3 - 2016

\author{
Margaret Czart \\ South University Online, USA
}

Correspondence: Margaret Czart, Online Programs in Health Science, South University Online, 8647 Sunset Rd, Niles, IL, USA, Email mczartl@gmail.com

Received: February 28, 2016 | Published: March 26, 2016

\section{Introduction}

The use of internet by the consumer for health information on obesity, weight management and other obesity related diseases continues to soar. The need for information has formed a health informatics sub-specialty referred to as Consumer Health Informatics. The American Medical Informatics Association defines Consumer Health Informatics as a field devoted to informatics from multiple consumer or patient views. This area includes patient-focused informatics, health literacy and consumer education to empower and engage patients to manage their own health. ${ }^{1}$

Obesity is just one of many health conditions where consumer education for empowerment is important to improve their own health. As weight management issue leading to obesity is a continuously growing health problem in United States and Canada. In 2012, the percentage of obese adults 18 and older was $27.7 \%$ in US nationally. Unfortunately, by 2014 the US percentage of obese adults increased to $28.9 \%$. The three states with the higher than the national average for obesity includes Mississippi (35.5\%), West Virginia (35.7\%) and Arkansas $(35.9 \%){ }^{2}$ The obesity trends in Canada are similar to those of the United States. In 2012, Statistics Canada has reported that $18.4 \%$ of Canadians aged 18 and older have been reported as being obese. The most recent Canadian statistics from 2014 has reported 20.2\% of Canadians aged 18 and older as obese. ${ }^{3}$ The Canadian providences with the higher than the national average obesity percentage include Newfoundland and Labrador (30.4\%), Nova Scotia (27.8\%) and New Brunswick (26.4\%). ${ }^{4}$
The 2014 obesity statistics based on self-reported data from both the United States and Canada continue to show that health literacy continues to be an issue. Although, in today's technological society there are additional new abilities and skills required for health literacy. Computer literacy which is defined as "an understanding of the concepts, terminology and operations that relate to general computer use and the essential knowledge needed to function independently with a computer". This computer literacy skill is required to assess, understand, and apply health information obtained through the internet. ${ }^{5}$ Being able to assess the information as to its reliability and accuracy is critical vs. assessing the sophisticated design of any given webpage. Individuals who lack computer literacy at an introductory level will face barriers in assessing information. Therefore, it is up to various health professionals to assist those who lack computer literacy skills in locating valuable resources to obtain health information relevant to their condition.

Currently, there is a large number of websites with information on obesity, healthy eating and weight management available to consumers on their personal computers, tablets and other mobile devices. Unfortunately, due to advances in technology there is a significant amount of webpages and mobile applications developed by third party developers that may not be interested in providing accurate or reliable health information. In addition, the more reliable resources by the US and Canadian government, non-profit organizations and healthcare organizations may not always come up as the top results of a general search in various web browsers (Google, Yahoo, Bing etc.). Therefore it is critical for various public health and other health 
professionals to bring more awareness as to reliable recourses for consumers. Consumers should be provided with guidelines when searching for health information on the internet. The US National Library of Medicine, Food and Drug administration and other organizations provide consumers with a good checklist to follow when searching the web for health information. The checklist falls into five main categories of:
a. Accuracy,
b. Authority,
c. Bias/Objectivity,
d. Currency/Timeliness and
e. Coverage. ${ }^{6}$

In my opinion, in effort to assist consumers searching for health in regards to health literacy and computer literacy skills the US government should promote and place more awareness on health information websites. As an average consumer may not be aware of websites such as MedlinePlus and Healthfinder which are intended for consumers and managed by US government agencies. ${ }^{7,8}$ Alternatively, various health professionals should recommend other specific reliable resources which consumers may visit for health information. Therefore, my recommendation for consumers is to follow the following steps when searching for health information:

a. Visit sites that end in ".gov," sponsored by the federal government

b. Visit ".edu" sites, which are run by universities, university hospitals or medical schools

c. Visit ".org" sites maintained by not-for-profit groups whose focus is research and teaching the public about specific diseases or conditions,

d. Speak with a professional regarding additional reliable resources and or terms to research.

\section{Conclusion}

In conclusion, though there are many sites whose addresses end in the ".com" extension only a small portion contains reliable and accurate information. A majority of these sites tend to commercial sites and are often selling products. Consumers searching for information regarding weight management or information regarding popular diets (Weight Watchers, South Beach Diet etc.) which may not be good for everyone. Therefore, it is important to follow the recommended steps to minimize any potential health risks from unreliable resources.

\section{Acknowledgements}

Paul Belanger, KFL\&A Public Health Unit, Ontario Canada

Kimberley Sears, Queen's University Healthcare Quality Program, Ontario Canada

\section{Conflict of interest}

The author declares no conflict of interest.

\section{References}

1. American Medical Informatics Association. Consumer Health Informatics. 2015.

2. Centers for Disease Control and Prevention. Nutrition, Physical Activity and Obesity: Data, Trends and Maps. 2016.

3. Statistics Canada. Overweight and obese adults (self-reported), 2012. 2015.

4. Statistics Canada. Overweight and obese adults (self-reported), 2014. 2015.

5. Bernhardt J, Brownfield D, Parker R. Understanding Health Literacy. In: Schwartzberg J, VanGeest J, et al. editors. Understanding Health Literacy Implications for Medicine and Public Health. USA: American Medical Association; 2005. p. 3-16.

6. Kelli Ham. Consumer Health \& Technology Coordinator, National Network of Libraries of Medicine, Pacific Southwest Region, Los Angeles, USA.

7. US National Library of Medicine. Medline Plus. 2016.

8. US Department of Health and Human Services. Health finder. 2016. 